\title{
Success prioritized distributed coordination function
}

\author{
Daisuke Umehara $^{1 \text { a) }}$, Hidekazu Murata ${ }^{2}$, and Satoshi Denno ${ }^{3}$ \\ ${ }^{1}$ Faculty of Information and Human Sciences, Kyoto Institute of Technology, \\ Matsugasaki, Sakyo-ku, Kyoto 606-8585, Japan \\ ${ }^{2}$ Graduate School of Informatics, Kyoto University, \\ Yoshida-honmachi, Sakyo-ku, Kyoto 606-8585, Japan \\ ${ }^{3}$ Graduate School of Natural Science and Technology, Okayama University, \\ 3-1-1 Tsushima-naka, Kita-ku, Okayama 700-8530, Japan \\ a)umehara@kit.ac.jp
}

\begin{abstract}
In this letter, we present a random access protocol with success priority, success prioritized distributed coordination function (SP-DCF), based on IEEE 802.11 DCF. Success stations (STAs) are prioritized contentionlessly to transmit the next data frame in the transmit queue in a random manner. We develop a performance analysis model of SP-DCF which enables us to estimate a variety of performance indexes such as the throughput or the frame discard rate (FDR) in a high accuracy for saturated wireless networks. The numerical analysis and simulation results show that the proposed SP-DCF achieves higher throughput and lower FDR as compared with the conventional DCF especially for heavily-congested wireless networks.
\end{abstract}

Keywords: IEEE 802.11 DCF, success priority, performance analysis model, consecutive successes, throughput, frame discard rate

Classification: Fundamental Theories for Communications

\section{References}

[1] IEEE Std 802.11-2012, "Part 11: Wireless LAN medium access control (MAC) and physical layer (PHY) specications," March 2012.

[2] IEEE Std 802.11ac-2013, "Amendment 4: Enhancements for very high throughput for operation in bands below $6 \mathrm{GHz}$," Nov. 2013.

[3] G. Bianchi, "Performance analysis of the IEEE 802.11 distributed coordination function," IEEE J. Sel. Areas Commun., vol. 18, no. 3, pp. 535-547, March 2000. DOI:10.1109/49.840210

[4] D. Umehara, H. Murata, and S. Denno, "Difference analysis in IEEE 802.11 DCF,” Proc. APSITT 2015, Colombo, Sri Lanka, pp. 55-57, Aug. 2015. DOI:10.1109/APSITT.2015.7217124

[5] B. A. H. S. Abeysekera, T. Matsuda, and T. Takine, "Dynamic contention window control mechanism to achieve fairness between uplink and downlink ows in IEEE 802.11 wireless LANs," IEEE Trans. Wireless Commun., vol. 7, no. 9, pp. 3517-3525, Sept. 2008. DOI:10.1109/TWC.2008.070304 
[6] D. Umehara, H. Murata, and S. Denno, "IEEE 802.11 DCF with successful transmission priority," Proc. ICSPCS 2015, Cairns, Australia, pp. 1-7, Dec. 2015. DOI:10.1109/ICSPCS.2015.7391794

\section{Introduction}

IEEE 802.11 distributed coordination function (DCF) is a distributed MAC protocol with the function of clear channel assessment (CCA) established by IEEE 802.11 standards $[1,2]$. Bianchi presented a simple and precise performance analysis model by using a two-dimensional Markov chain [3]. A station (STA) sends a frame without contention when the random backoff counter is allocated to zero after it sends the previous frame successfully in the IEEE 802.11 DCF originally developed in the last half of 1990s. The throughput will be higher due to the effect of this priority particularly when the minimum contention window is extremely small. Some of researches have been reported with respect to this phenomenon such as a modification of Bianchi's performance analysis model [4] and a prioritized access control for bidirectional traffic between an access point (AP) and a group of STAs [5].

In this letter, we propose a success prioritized DCF (SP-DCF), in which a success STA is more actively prioritized among all STAs in the wireless networks [6]. The priority of a success STA becomes high with increasing differential interframe space number (DiIFSN) between a success STA and all other deferred STAs. The success STA has an opportunity to contentionlessly transmit the next data frame so that it reduces the frame collision probability. As a result, the success priority enables us to enhance the throughput and reduce the frame discard rate (FDR). We also develop a performance analysis model of SP-DCF with high accuracy extended from Bianchi's performance analysis model.

\section{Success prioritized DCF}

Let us consider single-hop wireless networks with an AP and $n$ STAs where $n \geq 2$. AP does not have any data frames at STAs while STAs always have data frames at AP. Each STA has one wireless medium interface so that it cannot transmit a data frame and assess the channel simultaneously. AP and STAs are not hidden with each other.

\subsection{IEEE 802.11 DCF}

Let us describe the binary exponential backoff algorithm of IEEE 802.11 DCF. The backoff algorithm is conducted before the data frame transmission when the transmit queue is non-empty or the channel is detected busy during the DCF IFS (DIFS) time $T_{\text {difs }}$. A backoff counter $k$ is uniform-randomly selected from an integer set of $[0, \mathrm{CW}]$, where the contention window $(\mathrm{CW})$ is initialized to $\mathrm{CW}_{\min }=$ $W-1=W_{0}-1$ if it is the first time to transmit the data frame. The positive $k$ decrements by one when the channel is idle during the slot time $\sigma$. The decrease of $k$ is deferred when the channel is detected busy and it decrements by one when it 
observes an idle channel during $T_{\text {difs }}$. The STA starts transmitting the data frame after the decremented $k$ becomes 0 . AP correctly receives the data frame from a STA and then it transmits the ACK frame after elapsing the short IFS (SIFS) time $T_{\text {sifs. }}$. The data frame transmission is successful when the ACK frame is received at the source STA and $\mathrm{CW}$ is initialized to $\mathrm{CW}_{\min }$. It fails otherwise and the STA attempts to retransmit the same data frame with the updated $\mathrm{CW}=\min \{2(\mathrm{CW}+$ 1) $\left.-1, \mathrm{CW}_{\max }\right\}$ when the number of retransmissions is less than an integer of $r$, where $\mathrm{CW}_{\max }=2^{m}\left(\mathrm{CW}_{\min }+1\right)-1, m$, and $r+1$ stand for maximum contention window, maximum binary exponent, and retry limit, respectively. The number of retransmissions reaches $r$ and then the data frame is discarded when it fails and $\mathrm{CW}$ is initialized to $\mathrm{CW}_{\min }$. $\mathrm{CW}$ in the $i$-th transmissions of the same data frame for $i \in[0, r]$ is calculated as $\mathrm{CW}_{i}=W_{i}-1=2^{u_{i}}\left(\mathrm{CW}_{\min }+1\right)-1$ where $u_{i}=$ $\min \{m, i\}$.

\subsection{SP-DCF and its performance analysis model}

The success priority is established by alternating the IFS with the current STA state in the proposed SP-DCF. The IFS after transmitting the data frame successfully is called success IFS (SuIFS), $T_{\text {suifs, }}$, whereas the IFS after detecting the channel busy is called busy IFS (BuIFS), $T_{\text {buifs. }}$ The DiIFSN is defined as an integer of $D=$ ( $\left.T_{\text {buifs }}-T_{\text {suifs }}\right) / \sigma \in[0, W-1]$. The $D$ is positive in the proposed SP-DCF whereas it is equal to 0 in the conventional DCF. The next data frame after a success has a backoff counter $k$ uniform-randomly distributed over $\left[0, \mathrm{CW}_{\min }\right]$ so that it is consecutively transmitted without contention when $k$ is less than $D$. The probability of $m$ consecutive successes is expressed as $(1-D / W) \cdot(D / W)^{m-1}$ so that the average number of consecutive successes is derived as

$$
N_{\mathrm{S}}=\left(1-\frac{D}{W}\right) \sum_{m=1}^{\infty} m \cdot\left(\frac{D}{W}\right)^{m-1}=\frac{W}{W-D} .
$$

The average number of idle slots consumed during consecutive successes without contention is derived as

$$
\begin{aligned}
N_{\sigma} & =D+\frac{D-1}{2} \cdot\left(1-\frac{D}{W}\right) \sum_{m=1}^{\infty} m \cdot\left(\frac{D}{W}\right)^{m} \\
& =D \cdot\left(1+\frac{D-1}{2(W-D)}\right) .
\end{aligned}
$$

Let us define three variable-length slots which have the same backoff counter for channel-assessing STAs and they are classified into idle, success, and collision slots. Let us consider two-dimensional states of backoff stage $i$ and backoff counter $k$, and their state transitions. The collision slot probability $p$ by a frame transmission is assumed to be independent of its current backoff stage. The steady-state probability of a backoff state $(i, k)$ is denoted as $b_{i, k}$. The transmit probability $\tau$ by a slot is expressed as the summation of $b_{i, 0}$ over $i \in[0, r]$ and is derived as

$$
\tau(p)=\frac{2}{1-D \cdot(1-p)+W \cdot\left[\sum_{i=0}^{u-1}(2 p)^{i}+(2 p)^{u} \sum_{i=0}^{r-u} p^{i}\right] / \sum_{i=0}^{r} p^{i}},
$$


where $u=u_{r}$. The $p$ is expressed as $p=1-(1-\tau)^{n-1}$, i.e.

$$
\tau(p)=1-(1-p)^{\frac{1}{n-1}} .
$$

The values of $\tau$ and $p$ are derived by solving the non-linear system, composed of monotonically decreasing Eq. (3) with $\tau(0)=2 /(1-D+W)$ and strictly monotonically increasing Eq. (4) with $\tau(0)=0$ and $\tau(1)=1$. Since one or more successes are included in a success slot, the collision probability $p_{\mathrm{C}}$ by a frame transmission is expressed as

$$
p_{\mathrm{C}}=\frac{p}{N_{\mathrm{S}} \cdot(1-p)+p}=\frac{W-D}{W-p \cdot D} \cdot p,
$$

by compensating with $N_{\mathrm{S}}$.

The idle slot probability $P_{\sigma}$, the success slot probability $P_{\mathrm{S}}$, and the collision slot probability $P_{\mathrm{C}}$ by a slot are expressed as $P_{\sigma}=(1-\tau)^{n}, P_{\mathrm{S}}=n \tau(1-\tau)^{n-1}$, and $P_{\mathrm{C}}=1-P_{\sigma}-P_{\mathrm{S}}$, respectively. Let us denote the average data frame time as $T_{\text {data }}$ and the ACK frame time as $T_{\text {ack }}$. The propagation delay $\delta$ between any two STAs is assumed to be constant. The time consumed with a success of a single data frame is estimated as

$$
T_{\mathrm{S}}=T_{\text {data }}+T_{\text {ack }}+T_{\text {sifs }}+T_{\text {suifs }}+2 \delta .
$$

The time consumed with a collision of two or more data frames for channelassessing STAs is estimated as

$$
T_{\mathrm{C}}=T_{\text {data }}+T_{\text {buifs }}+\delta .
$$

The time consumed with a collision of two or more data frames for each transmitting STA is estimated as $T_{\text {data }}+T_{\text {ato }}+T_{\text {buifs }}$ where $T_{\text {ato }}=T_{\text {sifs }}+\sigma+T_{\text {prsd }}$ stands for ACK timeout and $T_{\text {prsd }}$ stands for the PHY-RX-START delay. Colliding, i.e. simultaneously transmitting STAs will be in a minority so that the collision slot time approximates $T_{\mathrm{C}}$ in the performance analysis model. $T_{\mathrm{S}}$ and $T_{\mathrm{C}}$ are easily extended to the request-to-send (RTS)/clear-to-send (CTS) access mode [3, 4, 6].

The throughput is expressed as

$$
S=\frac{P_{\mathrm{S}} \cdot N_{\mathrm{S}} \cdot M \cdot B}{P_{\sigma} \cdot \sigma+P_{\mathrm{S}} \cdot\left(N_{\sigma} \cdot \sigma+N_{\mathrm{S}} \cdot T_{\mathrm{S}}\right)+P_{\mathrm{C}} \cdot T_{\mathrm{C}}},
$$

where $B$ and $M$ stand for the average data MAC service data unit (MSDU) size and the average number of aggregated MAC protocol data unit (A-MPDU) subframes. Since the data frame is discarded when the ACK frame is not received even in the $r$-th retransmission, FDR is expressed as

$$
q_{\mathrm{fdr}}=\frac{p^{r+1}}{N_{\mathrm{S}} \cdot\left(1-p^{r+1}\right)+p^{r+1}}=\frac{W-D}{W-p^{r+1} \cdot D} \cdot p^{r+1} .
$$

\section{Numerical analysis and simulation results}

The parameters in the MAC layers of DCF and SP-DCF are illustrated in Table I. STAs do not detect any other data frames in the air during $T_{\mathrm{cns}}$ before their own frame transmissions. The propagation delay is assumed to be $\delta=0.1 \mu$ s. Let us employ $2 \times 2$ MIMO, 108 data subcarriers, 16-QAM, rate-1/2 LDPC code, and short guard interval (GI) of $0.4 \mu \mathrm{s}$. As a result, the PHY rate becomes $120 \mathrm{Mbps}$. 
Table I. The parameters in the MAC layers.

\begin{tabular}{|l|c|}
\hline \multicolumn{1}{|c|}{ Parameter } & Value \\
\hline Data MSDU size, $B$ & 1,500 bytes \\
\hline \# of A-MPDU subframes, $M$ & 10 \\
\hline ACK MPDU size & 32 bytes \\
\hline Slot time, $\sigma$ & $9 \mu \mathrm{s}$ \\
\hline SIFS time, $T_{\text {sifs }}$ & $16 \mu \mathrm{s}$ \\
\hline DIFS time, $T_{\text {difs }}$ & $34 \mu \mathrm{s}$ \\
\hline PHY-RX-START delay, $T_{\text {prsd }}$ & $25 \mu \mathrm{s}$ \\
\hline Carrier non-sensing time, $T_{\mathrm{cns}}$ & $4 \mu \mathrm{s}$ \\
\hline Minimum contention window, $\mathrm{CW}_{\text {min }}$ & 15 \\
\hline Maximum binary exponent, $m$ & 6 \\
\hline Retry limit, $r+1$ & 7 \\
\hline SuIFS time, $T_{\text {suifs }}$ & $16 \mu \mathrm{s}$ \\
\hline
\end{tabular}

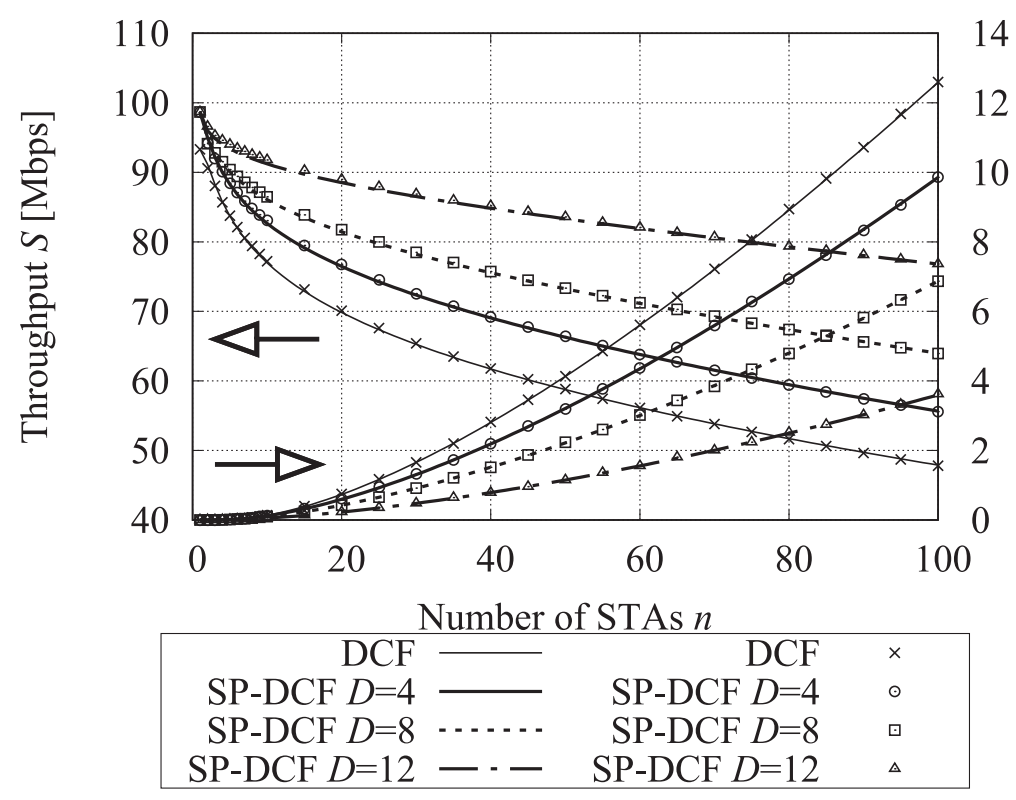
14

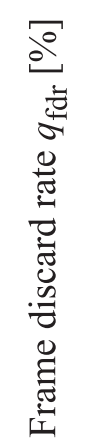

Fig. 1. Throughput and FDR versus the number of STAs.

Simulations including the effect of $T_{\text {cns }}$ and $T_{\text {ato }}$ are conducted to estimate throughput and FDR by using GNU Octave. They start on condition that the contention window of all STAs is set at $\mathrm{CW}_{\min }$ and terminate when $10^{6}$ data frames are successfully transmitted.

Fig. 1 illustrates the numerical analysis and simulation results with respect to throughput and FDR versus the number of STAs for DCF and SP-DCF with $D=4$, 8 , and 12. The numerical analysis results are drawn as lines whereas the simulation results are plotted as points, and both the results are agreed with each other in high accuracies. The throughput and FDR of SP-DCF is better than those of DCF and the differences become large as increasing $D$ and $n$. The simulation result for $n=100$ shows that the throughput of DCF is $47.8 \mathrm{Mbps}$ while that of SP-DCF with $D=12$ is $76.8 \mathrm{Mbps}$ so that SP-DCF enables to enhance the throughput of 
29.0 Mbps as compared with DCF. It also shows that the FDR of DCF is $12.6 \%$ while that of SP-DCF with $D=12$ is $3.6 \%$ so that SP-DCF is extremely effective for the reduction of FDR and it will be good matched with the behavior in the upper layers.

\section{Conclusion}

In this letter, we have developed SP-DCF and its performance analysis model. The numerical analysis and simulation results have agreed with each other in high accuracies and have shown that the proposed SP-DCF achieves higher throughput and lower FDR as compared with the conventional DCF especially for heavilycongested wireless networks.

\section{Acknowledgment}

This research and development work was supported by JSPS KAKENHI Grant Numbers JP15H04007, JP16K14268, and the MIC/SCOPE \#165007006. 